\title{
Las políticas de precios y reembolsos farmacéuticos en México, OCDE, 2007
}

\author{
Pierre Moïse, MA, (I) Elizabeth Docteur. ${ }^{(1)}$
}

\begin{abstract}
$C^{a}$ omo parte de sus actividades en políticas farmacéuticas, la Organización para la Cooperación y el Desarrollo Económico (OCDE) ha analizado los sistemas de precios y reembolsos de varios de sus países miembros. En el presente artículo se presentan extractos de uno de los documentos más recientes sobre políticas farmacéuticas, que fue publicado en $2007^{1}$ y que se titula Las políticas de precios y reembolsos farmacéuticos en México.

El gasto farmacéutico en México representa 21\% de su gasto total en salud, rebasando así el promedio de los países miembros de la OCDE. Dado el elevado gasto de bolsillo de los consumidores en ese país, existe preocupación sobre la sostenibilidad de proveer un acceso adecuado a los medicamentos. Debido a que estos últimos desempeñan un papel cada vez más importante en la atención de la salud, será cada vez más difícil sufragarlos como gastos de hogar. De manera similar será necesario incrementar la subvención pública si los mexicanos desean, al menos, mantener los niveles de consumo de fármacos y expandir la cobertura pública mediante el nuevo plan de seguridad social en salud denominado Seguro Popular, el cual permite el acceso a los servicios de salud a la enorme mayoría de ciudadanos que no cuentan con un seguro médico. Los productos farmacéuticos contribuyen significativamente a reducir la morbilidad y la mortalidad, y su gasto representa una
\end{abstract}

fracción sustancial del gasto en salud, todo lo cual les otorga gran importancia ante los encargados de tomar decisiones.

El medio en que se desenvuelve la política farmacéutica de México atraviesa por un período de rápida evolución, donde las políticas son desarrolladas, refinadas e implementadas de manera simultánea. Esto merma los esfuerzos del país para alcanzar eficientemente las metas de las políticas en la actualidad, y dificulta la evaluación misma de las políticas así como lograr una descripción precisa de ellas.

El gobierno mexicano reconoce la necesidad de contar con un marco de trabajo integrado y un esquema coordinado de políticas. Aunque continúa siendo controversial entre los expertos, los principales interesados y los funcionarios del sector farmacéutico nacional, un avance importante fue la publicación, en 2005, del documento titulado Hacia una política farmacéutica integral para México, elaborado por la Secretaría de Salud como una declaración de intención y dirección.

\section{Alcances y objetivos del estudio de la OCDE}

La publicación sobre las políticas de precios y reembolsos en México es parte del proyecto de la OCDE "Políticas farmacéuticas de precios e innovación", que tiene dos objetivos principales:

(I) División Salud, Dirección de Empleos, Mano de Obra y Asuntos Sociales, Organización para la Cooperación y el Desarrollo Económico, París, Francia. 
1. Contribuir a la base de información sobre políticas de precios de medicamentos en los países miembros, y desarrollar una taxonomía y un marco de trabajo para comparar políticas a nivel internacional.

2. Analizar y comparar los impactos e implicaciones de las políticas en los diferentes países, particularmente con respecto a su impacto en los precios de medicamentos en otros países, así como en actividades de investigación y desarrollo (I\&D) de la industria farmacéutica. ${ }^{1}$

En este informe específico sobre México, se analiza el ambiente político y las características del mercado del sector farmacéutico, así como en qué medida se han alcanzado algunas de las metas establecidas por las políticas farmacéuticas.

El documento se basa en una extensa revisión documental sobre las políticas de precios y reembolsos en México. Además, el equipo de autores de la OCDE entrevistó a los principales involucrados en este tema en México durante una visita de campo organizada en 2006, en colaboración con el personal de la Unidad de Análisis Económico de la Secretaría de Salud de México, la cual recomendó la lista de entrevistados con base en una lista de perfiles de actores que el equipo de la OCDE deseaba entrevistar. Los actores principales aportaron documentos e hicieron presentaciones verbales adicionales, todo lo cual revisó el equipo de la OCDE. Es importante mencionar que los perfiles de entrevistados fueron iguales en todos los países considerados en el estudio de la OCDE sobre políticas de precios y reembolsos, salvo el grupo de representantes de consumidores, los cuales no fueron entrevistados para el informe sobre México. Las siguientes secciones contienen extractos del texto completo del informe de la OCDE.

\section{Gastos}

En 2004 México gastó poco menos de 14 mil 400 millones de dólares (1.3\% de su PIB) en productos farmacéuticos, más que la mayoría de los países miembros de la OCDE. Sin embargo, al considerar los tamaños poblacionales, México ocupó el último lugar, con un promedio de sólo 138 dólares por habitante en 2004.

A diferencia de sus socios comerciales del TLCAN, en México los consumidores son la fuente directa de casi todo el gasto farmacéutico. Según la Recopilación de Información realizada conjuntamente por OCDEEurostat-OMS (SHA) en 2002, los mexicanos tienen un gasto de bolsillo por productos farmacéuticos considerablemente mayor que los consumidores de otros países. En 2003, 88\% del gasto total en productos farmacéuticos en México correspondió a gastos de bolsillo, a diferencia de Corea donde fue de $57 \%$, siendo esta última la única nación donde el gasto de bolsillo superó a la mitad del gasto total en productos farmacéuticos.

Con base en las estimaciones de la Secretaría de Salud (SSA), el volumen total de ventas farmacéuticas en México se divide en partes iguales entre los mercados público y privado. ${ }^{1}$ Sin embargo, el predominio de medicamentos de patente en el mercado privado y, por el contrario, de medicamentos genéricos en el mercado público, constituye la razón de la enorme discrepancia entre ambos mercados con respecto al valor de las ventas. De acuerdo con las estimaciones de la SSA, ${ }^{1}$ las ventas se distribuyen a razón de $82 / 18 \%$ del total entre los mercados privado y público.

El Instituto Mexicano del Seguro Social (IMSS) y el Instituto de Seguridad y Servicios Sociales de los Trabajadores del Estado (ISSSTE) representan, respectivamente, alrededor de 80.3 y $14.2 \%$ del gasto público en productos farmacéuticos, mientras que las otras instituciones de seguridad social y los servicios de salud estatales y federales representan tan sólo $5.4 \% .{ }^{2}$ El gasto del sector público en productos farmacéuticos, expresado como porcentaje del gasto total en dichos productos, fue de $8.2 \%$ en $2002,10.4 \%$ en 2003 y $11.6 \%$ en $2004 .{ }^{3}$

\section{Precio}

Según los cálculos de la Unidad de Análisis Económico de la SSA, existe una dicotomía en los precios farmacéuticos que refleja la participación de los mercados público y privado en el sistema de atención a la salud. Se estima que los precios de compra de los productos farmacéuticos en el sector público son, en promedio, aproximadamente de $1 / 4$ a 1/3 más bajos que en el mercado privado. ${ }^{4}$ Los cálculos realizados sobre una muestra de 52 medicamentos indican que los precios de compra en el IMSS eran 83\% más bajos que los precios actuales (observados) de menudeo en el sector privado.

Los datos recopilados por el IMSS sobre el valor y el volumen de los medicamentos vendidos en el sector privado pueden ayudar a esclarecer los precios de compra de los productos farmacéuticos en dicho sector. ${ }^{5} \mathrm{De}$ 1997 a 2002, el volumen de medicamentos vendidos en el sector privado permaneció básicamente sin cambios, mientras que su valor total se duplicó. Esto sugiere que los precios de los productos farmacéuticos en el sector privado aumentaron, tal como lo hicieron los precios en general. Sin embargo, un incremento en el valor de los medicamentos vendidos, con un volumen de ventas constante, también puede deberse a la sustitución de medicamentos baratos por otros más costosos. La imposición de nuevas leyes de derechos de propiedad 
intelectual, así como cambios en el perfil epidemiológico podrían haber conducido a tal sustitución. De hecho, los funcionarios del IMSS mencionaron los cambios en la epidemiología de sus derechohabientes, con modificaciones marginales en los patrones de consumo, como una causa de la transición hacia la compra de medicamentos más costosos por parte del IMSS.

Datos compilados por el Banco Central de México muestran una evolución de la inflación en los precios al consumidor y en los precios de productos farmacéuticos entre los años 1988 y 2005, período en el que hubo varios cambios en los reglamentos sobre precios en México. De 1988 a 1990 la inflación en los precios al consumidor fue mayor que la correspondiente a los precios de productos farmacéuticos, en una época en la que aún prevalecían los estrictos controles de precios establecidos en 1984. Cada año, a partir de 1991 cuando iniciaron las negociaciones entre el gobierno y la industria farmacéutica para reemplazar los controles estrictos por otros más flexibles, los precios de los farmacéuticos han aumentado a un ritmo más acelerado que los precios al consumidor en general. En el lapso comprendido entre el comienzo de las negociaciones entre el gobierno y la industria y la firma del convenio de normatividad de precios en 1996, las tendencias inflacionarias de los precios farmacéuticos y los precios al consumidor se bifurcaron y la inflación en los precios farmacéuticos cayó de 35.8\% en 1996 a 7.2\% en 2005.

Desde un punto de vista histórico, los precios de productos farmacéuticos en México han sido bajos en comparación con los de otros países miembros de la OCDE. A principios de la década de 1990, se estimaba que los precios en México eran más o menos cinco veces inferiores a los de los Estados Unidos (EUA) y tres veces menores a los de Europa. ${ }^{6}$ Desde entonces, los gobiernos sucesivos han promovido iniciativas para liberar las importaciones, fortalecer los derechos de propiedad intelectual (DPI) y hacer más laxos, sin por ello eliminar, los controles de precios, estrechando así la brecha entre los precios en México y aquellos de los otros países miembros de la OCDE.

Sin embargo, Danzon y Furukawa ${ }^{7}$ encontraron que cuando los precios se ajustan de acuerdo al ingreso, las diferencias de los precios farmacéuticos entre países reflejan, a grandes rasgos, las diferencias en ingresos, salvo en los casos de México y Chile, donde el nivel de los precios farmacéuticos resulta ser más de cinco veces el nivel de precios ajustados al ingreso en los EUA. En tanto que el ingreso puede considerarse como un indicador aproximado de la elasticidad de los precios ante la demanda de productos farmacéuticos, los mexicanos están pagando precios mucho más elevados de lo que pudiera esperarse, considerando su relativo bajo nivel de ingresos. Como señalan los autores antes mencionados, en el caso de México es posible que los niveles de precios reflejen las decisiones de los productores de no ofrecer precios más acordes con el ingreso por habitante, que se usa como un indicador aproximado de la elasticidad de la demanda, por temor a que sus productos sean importados por los EUA. Sin embargo, es cuestionable el uso del nivel de ingreso como un indicador aproximado de la elasticidad de precios ante la demanda.

Una comparación entre los precios farmacéuticos en México y en otros países de América Latina, con niveles similares de poder adquisitivo, demuestra que los precios en México son relativamente altos. De acuerdo con una muestra tomada en 2003 de precios promedio de medicamentos ofrecidos al público en México, Argentina, Brasil, Chile y Venezuela, se encontró que los precios en México son los más elevados. ${ }^{5}$

\section{Competencia}

La competencia entre fabricantes de productos farmacéuticos en el mercado público es mayor que en el privado. En el primero, 300 productores, en su mayoría nacionales, compiten para abastecer de medicamentos a las instituciones de seguridad social y a los servicios de salud estatales y federales. Las compañías multinacionales basadas en investigación están asociadas con alrededor de 20\% del valor total de los productos farmacéuticos que se venden en el mercado público. El mercado privado está mucho más concentrado, con cerca de 70 empresas que compiten en la venta de medicamentos. Aquí, la participación en el mercado se invierte, pues las compañías multinacionales representan $80 \%$ del valor total de ventas y las nacionales $20 \%$.

\section{Productos genéricos intercambiables}

Hasta hace poco tiempo los fabricantes de productos genéricos no estaban obligados a demostrar la bioequivalencia de un producto con respecto a su medicamento de referencia. Sin embargo, dos desarrollos recientes cambiarán esta situación. Un cambio reciente al Artículo 376 de la Ley General de Salud estipula que la aprobación de medicamentos para el mercado estará limitada a un período de cinco años; una vez expirado, el productor podrá solicitar la renovación y continuar haciéndolo cada cinco años a partir de entonces. El segundo desarrollo requiere que todos los productores sometan pruebas de bioequivalencia de sus productos genéricos como parte del proceso para obtener la aprobación para el mercado, incluyendo solicitudes para renovar la autorización de comercialización. Estos desarrollos eliminarán 
efectivamente todos los genéricos no bioequivalentes del mercado mexicano para el año 2010.

Desde 1998 el gobierno ha concentrado sus esfuerzos en promover el uso de productos genéricos bioequivalentes o intercambiables (recuadro 1). En apoyo a esos esfuerzos, en 2002 el Consejo de Salubridad General (CSG) emitió un mandato requiriendo que todas las instituciones de salud del sector público adquirieran productos genéricos intercambiables (GI) cuando estuvieran disponibles. El resultado ha sido un incremento en el número de GI enlistados, de $963^{8}$ a 3685 productos hasta julio de 2000, de un total de 7000 productos en el mercado. ${ }^{5}$ Actualmente existen 143 fabricantes participantes y 27 laboratorios contratistas (terceros-autorizados) para realizar exámenes de bioequivalencia.

La promoción de GI por parte del gobierno también ha dado resultados mixtos en términos de la prescripción médica de estos productos. Casi la mitad de todas las prescripciones en el sector público fueron de genéricos, lo cual se compara favorablemente con países con una alta penetración de este tipo de productos. Sin embargo, en el mercado privado la penetración de genéricos en prescripciones es aún escasa (menos de $5 \%$ de las prescripciones), mientras que el número de genéricos no-intercambiables en el mercado se ha mantenido en crecimiento. Se estima que en $60 \%$ de los medicamentos

\section{Recuadro I \\ DifERENCIACIÓN DE PRODUCTOS FARMACÉUTICOS EN EL MERCADO MEXICANO}

Producto original. La primera versión de un producto medicinal, desarrollado y patentado por la compañía farmacéutica que lo creó y que recibió el derecho exclusivo para comercializar el producto durante un período específico. Un producto original es un medicamento de marca vendida por la compañía que la creó o por otra que haya recibido de ésta una autorización o licencia, esté o no patentado dicho producto. Estos productos se conocen comúnmente comúnmente como medicamentos "innovadores" o "de referencia".

Producto genérico intercambiable (Gl). Desde 1998 una versión genérica de un producto original que ha perdido su patente, puede certificarse como bioequivalente e intercambiable con un producto de referencia.

Producto genérico no intercambiable (similar). Una versión genérica de un producto farmacéutico original, que no ha sido probada como bioequivalente a su producto de referencia ni como intercambiable con él desde el punto de vista terapéutico.

Producto copia. Medicamento aparentemente bioequivalente a, e intercambiable con, un producto de referencia patentado en otro país.A partir del refuerzo de los derechos de propiedad intelectual en los años noventa, su número se ha reducido progresivamente. actualmente en el mercado no se han presentado pruebas de bioequivalencia. ${ }^{5}$ Los genéricos no-intercambiables, $\mathrm{o}$ similares, representaban aproximadamente $12 \%$ del total de ventas farmacéuticas al público. ${ }^{9}$ Como se mencionó anteriormente, los similares se eliminarán eventualmente en los siguientes cinco años.

\section{Contención de los gastos en medicamentos}

A pesar de que el gasto total en salud de México ha crecido a un ritmo más lento que el promedio de los países de la OCDE, la tasa de crecimiento del gasto en productos farmacéuticos es una de las más altas de la OCDE. Esto podría explicarse, en parte, por el efecto del fuerte incremento en los precios de menudeo de medicamentos de patente, lo cual refleja una relajación en los controles de precios farmacéuticos en el mercado privado en México.

Actualmente la regulación mexicana de precios no es eficiente para la restricción de los precios de los productores, y las políticas para el control del volumen o consumo son prácticamente inexistentes en el sector privado, lo cual deja a la mitad del sector farmacéutico en México esencialmente no regulado. Dado que los precios de menudeo en México son muy altos con respecto al nivel de ingresos, la tercera parte de todo el gasto privado en salud se destina a productos farmacéuticos.

Por otro lado México ha logrado, con bastante éxito, contener el crecimiento del gasto en medicamentos en el sector público potenciando su poder adquisitivo para obtener precios muy bajos y estableciendo, al mismo tiempo, controles estrictos sobre el abastecimiento de farmacéuticos a aquéllos con cobertura pública de seguro médico, lo cual también trasfiere parte de la demanda (y de los costos) de este rubro al sector privado. Como reflejo de estos factores, el gasto farmacéutico representó sólo 5.2\% del gasto público total en salud en México en 2004, que representa un porcentaje notablemente bajo según los estándares de la OCDE.

A medida que se extienda la cobertura pública de seguros médicos en México, aumentará la presión sobre los costos en el sector público. No está claro si los productores continuarán proporcionando productos a precios tan bajos a una proporción mayor del mercado total. Más aún, en la medida en que los productores teman que las restricciones del empaquetado lleguen a facilitar la reventa ilícita en el mercado privado de productos fabricados para compradores en el sector público, estarán menos dispuestos a vender sus productos al público con un descuento significativo. 


\section{Sostenibilidad y equidad del financiamiento de productos farmacéuticos}

En la medida en que adquiera cada vez más importancia el papel de los productos farmacéuticos en el cuidado de la salud y en el gasto total en salud, más importante para México será también abandonar la dependencia del gasto de bolsillo. Depender del gasto de hogar como la fuente principal de financiamiento es insostenible y hace necesario incrementar la cobertura pública o los seguros médicos privados, lo cual mejora tanto la sostenibilidad como la equidad y, al mismo tiempo, ofrece las ventajas de compartir los riesgos y de reducir las barreras financieras a los servicios. Sin embargo, dada la extensa proporción de mexicanos que no cuentan con empleo formal, las implicaciones financieras de expandir la cobertura financiada públicamente son muy significativas. Adicionalmente, se puede esperar que el incremento de la cobertura tenga un fuerte impacto positivo en la demanda de farmacéuticos, como parece ser el caso de los servicios de salud en general. ${ }^{10}$

\section{Eficiencia de los gastos}

La evidencia sugiere que los gastos farmacéuticos en México no conducen a los resultados más costo efectivos. Actualmente, los principales esfuerzos por obtener un valor óptimo en el gasto en medicamentos se realizan en el campo de las decisiones de inclusión en el Cuadro Básico y en el Catálogo de Insumos, y las subsecuentes decisiones sobre adquisiciones por esquemas de cobertura financiados públicamente. No obstante, el uso del análisis económico en tales decisiones es relativamente nuevo y no existen normas para éste.

Las mejoras en la eficiencia podrían incluir el reemplazo del modelo de compras por uno de reembolso a pacientes o farmacias por concepto de gastos en medicamentos, dados los costos asociados con la compra, el almacenamiento y la distribución de los medicamentos a pacientes con cobertura financiada públicamente. Por otra parte, si bien el hecho de incrementar la correspondencia entre necesidad y disponibilidad mejoraría la eficiencia, también podría resultar en costos más elevados.

En México los medicamentos genéricos tienen una participación importante en el mercado en general, particularmente en el público. Empero, la efectividad puede ser comprometida innecesariamente en el caso de depender de medicamentos cuya bioequivalencia no ha sido probada. Si la alta participación en el mercado puede mantenerse a la luz de las demandas de bioequivalencia, México podría jactarse en particular de tener un mercado público costo-efectivo, aunque la presencia significativa de genéricos no bioequivalentes podría socavar lo que debería ser un abastecimiento costo efectivo de farmacéuticos.

En el aspecto privado, la falta de competencia en el mercado de mayoreo podría ser una fuente de ineficiencia. Sin embargo, el abasto insuficiente no es evidente, la fijación de precios máximos limita la especulación, y falta evidencia que indique la existencia de distribución ineficiente. A lo largo de la cadena de abastecimiento las farmacias pequeñas son las más propensas a pagar el precio de un mercado de mayoreo no-competitivo, exceptuando nuevamente las áreas rurales atendidas por un mayorista único.

\section{Disponibilidad de productos farmacéuticos}

Varios factores sugieren que la mayoría de los productos farmacéuticos disponibles en los países desarrollados también se consiguen oportunamente en México. En primer lugar, se informa que los períodos de aprobación son cortos. En segundo lugar, desde la perspectiva de las ventas México es uno de los mercados farmacéuticos más grandes del mundo, con un importante potencial de crecimiento. Para lograr las metas de ventas globales de las compañías, de las cuales dependen las ganancias, es razonable pensar que son pocos los fabricantes de fármacos que pueden darse el lujo de no hacer negocios en México. Aún así, las decisiones sobre el momento oportuno para el lanzamiento de productos podrían bien estar afectadas por la cercanía de México con los EUA y por el potencial del intercambio comercial a través de la frontera.

Aunque la evidencia confirma la existencia de una pronta disponibilidad de nuevos productos en México, es muy probable que ésta varíe significativamente a través del país. La variabilidad geográfica en el abastecimiento también es importante. De manera similar, la disponibilidad para los beneficiarios de seguridad social y de los seguros de salud financiados por fuentes públicas es distinta. No está claro si los medicamentos registrados en el Cuadro Básico y en el Catálogo de Insumos se encuentran permanente y fácilmente accesibles en las clínicas públicas, lo cual reflejaría factores tales como restricciones por costos y deficiencias en la planeación de la compra de medicamentos.

Dado que la disponibilidad de genéricos no intercambiables disminuirá en los próximos años, a medida que entren en vigor las normas que hacen necesaria la bioequivalencia, puede esperarse un incremento en el precio promedio global de medicamentos, lo cual acentuará los problemas de las personas con bajos ingresos. Por el contrario en la medida en que los genéricos intercambiables puedan sustituir a los medicamentos 
de patente más costosos, su amplia presencia podría contribuir a la reducción general de los precios de los medicamentos. Aun está por verse la manera en que el desplazamiento gradual de productos no intercambiables por genéricos intercambiables afectará a los precios de los medicamentos en general.

\section{Demanda de productos farmacéuticos}

De acuerdo con varios criterios de evaluación del estado de la salud, México está en peor situación que la mayoría de los países miembros de la OCDE, ceterus paribus, esto debería significar que la demanda de servicios de salud, incluyendo los farmacéuticos, debería ser mayor en México. Es claro que un factor limitante es el bajo ingreso per cápita en el país. Por otra parte, si los productos farmacéuticos son un bien necesario, entonces se esperaría que el gasto en estos productos absorbiera una proporción mayor del ingreso per cápita en México que en la mayoría de los otros países. Sin embargo, México sólo gasta 1.32\% de su PIB per cápita en fármacos, menos que el promedio de la OCDE; en consecuencia, los mexicanos podrían no están recibiendo todos los medicamentos que requieren.

\section{Calidad de servicio}

Al parecer en México no existen políticas diseñadas para afectar el uso de medicamentos por parte de pacientes, ni para buscar influir en los hábitos de dispensación de los empleados de farmacias. Ahí donde existen políticas que deberían influir sobre el consumo de productos farmacéuticos, resulta que están enfocadas para influir sobre las prácticas de prescripción de los médicos.

En México, la sustitución por genéricos es la política más ampliamente ejercida para influir en las prácticas de prescripción de los médicos. En el sector público, el Cuadro Básico y el Catálogo de Insumos enlistan medicamentos por sus nombres internacionales no patentados, incluyendo los productos originales. El IMSS distribuye una copia del Cuadro Básico a sus médicos lo cual, desde el punto de vista de esa institución los hace más conscientes de escribir el nombre genérico cuando prescriben medicamentos a sus pacientes. El IMSS también ha producido lineamientos para el tratamiento de 42 enfermedades comunes; si bien no están directamente orientados a influir sobre las prácticas de prescripción de los médicos, sí contienen directrices que enfatizan el uso de productos genéricos.

Los institutos de seguridad social del sector público promueven activamente el uso de productos genéricos intercambiables. Esta política puede considerarse bastante exitosa en cuanto a que el sector público adquiere enormes cantidades de estos productos. Sin embargo, esto podría conducir inadvertidamente a que gran cantidad de beneficiarios paguen por sus medicamentos de su bolsillo en farmacias de menudeo, dado que muchos consideran que los productos genéricos son de menor calidad.

\section{Satisfacción del público con las políticas y los resultados farmacéuticos}

Se dispone de muy poca información para evaluar la satisfacción de los pacientes y los consumidores con los resultados de la política farmacéutica en México. Anecdóticamente, existe una percepción de mala calidad de los productos farmacéuticos y de los servicios en el sector público. La solución propuesta por el gobierno, consistente en obligar a los fabricantes del sector público a vender sus productos en empaques idénticos a los del mercado privado, puede ser un arma de doble filo en el sentido de que las percepciones de calidad puedan mejorar inclusive cuando el acceso se vuelva más restringido (debido a las probables reacciones de los fabricantes a la imposición de cambios en el empaque).

Es muy probable que en el mercado privado exista una dicotomía de percepción entre la calidad real y la calidad percibida. Por ejemplo, los genéricos no intercambiables continúan siendo muy fuertes en México. Empero, esos productos (que son similares pero no necesariamente idénticos a los productos conocidos fabricados por las compañías desarrolladoras) pueden acarrear un mayor riesgo de ser ineficientes o inseguros que los genéricos bioequivalentes o los productos originales.

\section{Investigación y desarrollo}

México todavía carece de los recursos para atraer a los fabricantes farmacéuticos de la esfera de la industria que se basa en investigación y desarrollo. El intercambio comercial puede haber crecido, pero la desventaja comparativa de México en la fabricación de productos finales es evidente: en la actualidad $90 \%$ de las exportaciones farmacéuticas mexicanas son materias primas, mientras que las importaciones de productos finales han crecido en 50\% del total de las importaciones, cuando anteriormente eran de menos de $10 \%$. En cuanto a innovación, el país también se encuentra bajo presión por competir. Los niveles de financiamiento de actividades de investigación y desarrollo son bajos de acuerdo con los estándares de la OCDE e inclusive han disminuido en años recientes. El gobierno financia la mayor parte de la investigación en México, pero sin incrementos en los retornos habrá poco espacio para expandir el 
financiamiento de dichas actividades en vista de otras demandas en competencia. El financiamiento de la investigación y el desarrollo por parte de la iniciativa privada no están en mejor situación; es más, la cifra de investigadores es significativamente inferior a la de otros países de la OCDE. Las mejores expectativas para la industria farmacéutica mexicana se ubican en la posibilidad de utilizar su ventaja comparativa sobre otros países latinoamericanos: el intercambio comercial abierto y la fuerza de sus derechos de propiedad intelectual. La competitividad introducida por la apertura comercial obligará a los productores de genéricos a ser más eficientes y los requerimientos de bioequivalencia mejorarán la calidad de sus productos respecto a sus competidores latinoamericanos. En estas condiciones, la industria de genéricos en México está en posición de competir favorablemente con las industrias de otros países de la OCDE. Adicionalmente, la fuerza de los derechos de propiedad intelectual obligará a las compañías multinacionales a considerar a México como una base para América Latina.

\section{Evaluación del logro de las metas de las políticas}

Por último, no cabe duda que hoy en día los medicamentos son subutilizados en México, en el sentido de que muchas personas que podrían mejorar su salud por medio de medicamentos no pueden conseguirlos. El impacto de dicha subutilización no puede cuantificarse.

Según los estándares de la OCDE, el estado de la salud en México es muy bajo. Sin duda las mejoras en las políticas farmacéuticas elevarán el estado de salud de muchos mexicanos, pero como sucede con todas las políticas, existen tanto beneficios como costos, lo cual implica que se tendrían que hacer algunos sacrificios para que se produzcan los beneficios (trade-off).

En México, debido a la laxitud en el control de los precios de productos farmacéuticos en el mercado privado, y al crecimiento de los precios al menudeo de medicamentos de patente, el gasto en productos farmacéuticos ha aumentado hasta convertirse en una de las tasas más elevadas de la OCDE. La eficiencia debe incrementarse para garantizar la sustentabilidad y la equidad, mismas que podrían mejorarse apoyándose en mayor grado en la cobertura pública, o bien en los segu- ros privados. Esto ofrecería las ventajas de compartir los riesgos y reducir las barreras financieras para la atención a la salud. Para impulsar un mercado de productos altamente costo efectivos es necesario mantener una activa participación de los genéricos intercambiables en el mercado y alcanzar una elevada participación de estos genéricos en el mercado privado.

Las políticas para mejorar la calidad de los servicios de salud se han enfocado estrechamente, principalmente en influir sobre las prácticas de prescripción de los médicos en el sector público, tal como en el IMSS.

Hasta ahora, la fuerza de los derechos de propiedad intelectual no ha estimulado las actividades de investigación y desarrollo en México, lo cual es un indicador del bajo nivel de financiamiento y del reducido número de investigadores, en comparación con lo que sucede en otros países de la OCDE. Es necesario ponderar cuidadosamente los beneficios de la fuerza de los derechos de propiedad intelectual contra sus implicaciones en los precios y en el acceso a medicamentos.

\section{Referencias}

I. Moise P, Docteur E. Pharmaceutical pricing and reimbursement policies in Mexico. Health Working Paper. Paris: OCDE, 2007.

2. González-Pier E, González-Hernández A. Regulación saludable del sector farmacéutico. En: Comisión Federal de Competencia. Competencia Económica en México. México, DF: Comisión Federal de Competencia, 2004, capítulo X. 3. OCDE. OCDE health data 2006, Paris: OCDE. [Consultado el 26 de junio de 2006]Disponible en: http://www.OCDE. org/health/healthdata/

4. Gonzalez J. Industry Sector Analysis (Mexico). Washington, DC: US \& Foreign Commercial Service, 2002.

5. Ministry of Health. Hacia una política farmacéutica integral para México. México, DF: Secretaria de Salud, 2005.

6. IMS Health. IMS World Review, the Pharmaceutical Market, 1995.

Londres: IMS Health, 1995.

7. Danzon PM, Furukawa MF. Prices and availability of pharmaceuticals: evidence from nine countries. Health Aff 2003;Web Exclusive:W3-52I -W3-526. [Consultado el II de julio de 2006]. Disponible en: http:// content.healthaffairs.org/cgi/reprint/hlthaff.w3.52 Ivl, 8. OCDE. OCDE Review of Health Systems. México. Paris: OCDE, 2005. 9. IMS Health. Intelligence 360: Global Pharmaceutical Perspectives 2005 IMS Health, 2005. [Consultado el 22 de mayo de 2006]. Disponible en: http://www.imshealth.com/vgn/images/portal/cit_40000873/5/55/7484654 7Interface\%20APRIL\%202005.pdf

10. Gakidou E, Lozano R, González-Pier D,Abbott-Klafter J, Barofsky JT, Bryson-Cahn C, et al. Assessing the effect of the 200I-2006 Mexican health reform: an interim report card. Lancet 2006; 368: 1920-1935. 